\title{
Frequency and number of B-lines using a regionally based lung ultrasound examination in cats with radiographically normal lungs compared to cats with left-sided congestive heart failure
}

\author{
Gregory R. Lisciandro ${ }^{1, *}$, DVM, DABVP, DACVECC ; Robert M.Fulton ${ }^{2}$,DVM; Geoffrey T. \\ Fosgate $^{3}$, DVM, PhD, DACVPM and Kelly A. Mann ${ }^{4}$, DVM, DACVR \\ ${ }^{1}$ Hill Country Veterinary Specialists, Spicewood, TX \\ ${ }^{2}$ Veterinary Emergency and Specialty Center, Richmond, VA \\ ${ }^{3}$ Department of Research and Innovative Support, University of Pretoria, Gauteng, South Africa ( \\ ${ }^{4}$ Department of Environmental and Radiological Health Sciences, Colorado State University, Fort Collins, CO \\ *Address correspondence and offprint requests to: Dr. Gregory R. Lisciandro, 1049 Lakeshore Drive, Spicewood, \\ TX 78669, USA \\ Email: FastSavesLives@gmail.com
}

Conflicts of Interest: Dr. Lisciandro is the owner of FASTVet.com, a private corporation that provides veterinary ultrasound training to practicing veterinarians. He also teaches ultrasonography courses for EI Medical, Sound, SonoSite, and scil animal care company, and has received ultrasound equipment on loan from SonoSite, EI Medical, scil animal care, and Sound. Dr. Fulton also teaches ultrasonography for Sound. 


\section{Abstract}

Objective - To establish a baseline lung ultrasound artifact profile (dry lung [A-lines with glide sign] and wet lung [ultrasound lung rockets, ULRs, also called B-lines]) using Vet BLUE (Veterinary Bedside Lung Ultrasound Exam) in cats without clinical signs of respiratory disease and with radiographically normal lung findings and compared to a cohort with left-sided congestive heart failure (CHF).

Design - Prospective case series.

Animals - 49 cats without clinical signs of respiratory disease and with radiographically normal lungs and 7 cats with radiographical evidence of left-sided CHF.

Interventions - Application of an 8-view regionally-based lung ultrasound examination named Vet BLUE. Frequency of ULRs were statistically compared based on signalment, body condition score, investigator and reasons for radiography and between the 49 cats with radiographically normal ungs to 7 cats with radiographical evidence of left-sided CHF.. Results - Overall frequency of ULRs was $12 \%$ (95\% confidence interval, 5-24\%) in cats without respiratory disease versus $100 \%$ (95\% confidence interval, 65-100\%) in those with left-sided CHF. Six cats (6/49) had ULRs detected during Vet BLUE with 5/6 having ULRs at a single site; and 4/5 having a single ULR at 1 site, 1/5 having 2 ULRs at 1 site; and the sixth cat having 2 positive sites with single ULRs at each. In the cohort of cats with left-sided CHF, all cats (7/7) had $>3$ URLs detected at every site.

Conclusions - The predominance of dry lung artifacts in cats without respiratory disease and with radiographically normal lungs and the predominance of wet lung artifacts in cats with leftsided CHF suggests that Vet BLUE is clinically useful for the identification and evaluation of feline respiratory conditions. 


\section{Introduction}

Lung ultrasound is an important screening test and monitoring tool for the diagnosis and management of humans and dogs in respiratory distress or with respiratory disease. ${ }^{1,2,3,4,5}$ In humans, it has a higher sensitivity than lung auscultation and supine chest radiography for many acute and potentially life-threatening respiratory conditions. ${ }^{1,2}$ Lung ultrasound is a rapid (<2 minutes), safe, non-ionizing, point-of-care modality that differentiates common respiratory conditions without the delay in waiting for radiographs. ${ }^{1,5-10}$ The use of lung ultrasound increases the probability of an accurate working diagnosis, improving patient care from both a treatment and diagnostic standpoint. ${ }^{4,5,6}$

The fundamentals of lung ultrasound focus on the pattern-based observation of readily teachable dry lung (A-lines with a glide sign [normal]) and wet lung (ultrasound lung rockets or B-lines [interstitial infiltrate]) artifacts.$^{3-11,12}$ In radiographically normal human and canine lungs, ultrasound lung rockets (ULRs) are infrequent and dry lung artifacts predominate. ${ }^{1,2,4,5}$ Thus, when wet lung artifacts predominate, their frequency and regional distribution can be used in addition to traditional means of lung auscultation and thoracic radiography for ruling in and ruling out many causes of respiratory distress including asthma, chronic obstructive pulmonary disease, left-sided CHF, pneumonia, and pulmonary thrombo-embolism. ${ }^{1-3,8-11,13}$ Moreover, the use of the dry lung versus wet lung concept has been shown to reliably rule out or support the presence of pulmonary edema associated with left-sided CHF, better answering the historically difficult clinical question of whether dyspnea is due to pulmonary disease, left-sided CHF, or non-respiratory look-a-likes. ${ }^{1,2,5-7,9,10}$

Numbers of ULRs or B-lines (wet lung artifacts) correlate with the degree of interstitial and alveolar infiltrates in human lungs. ${ }^{2}$ Counting the numbers and locations of ULRs during a 
lung ultrasound exam provides semi-quantitative data regarding the presence and severity of pulmonary infiltrates. Serial exams may document a worsening (increasing ULR numbers) or improvement (decreasing ULR numbers) in the patient's condition, helping to guide therapy. ${ }^{1,5-}$ 7,9,13-15 Additional lung ultrasound findings associated with the severity of interstitial-alveolar consolidation have also been defined (shred sign, tissue sign, nodule sign $)^{12,14,15}$ but are not expected to be present in cats with radiographically normal lungs and were not observed in the analogous Vet BLUE study in dogs. ${ }^{4}$

The purposes of this study using Vet BLUE were to (1) establish a baseline frequency and number of ULRs in cats without clinical signs of respiratory disease and with radiographically normal lung findings; (2) identify significant predictors for the presence of ULRs in cats without respiratory signs and with radiographically normal lung findings; and (3) compare findings in normal cats to the frequency and number of ULRs in a cohort of cats with left-sided CHF.

We hypothesized that ULRs would be uncommon in cats without respiratory disease and with radiographically normal lung findings but frequent in cats with left-sided CHF as observed in an analogous study in dogs. ${ }^{4}$ Furthermore, we expected that there would be no effect of age, sex, breed, body weight, body condition score, investigator and reasons for thoracic radiography on the presence/absence of ULRs; and that other described lung ultrasound artifacts representing severe pulmonary interstitial-alveolar infiltration or consolidation would be absent.

\section{Materials and Methods}

Cats without clinical signs of respiratory disease presenting at 3 private veterinary referral and emergency centers were prospectively enrolled over a 37-month period (September 
2010- October 2013) after obtaining informed consent from owners. All cats underwent the Vet BLUE examination unsedated and in sternal or standing position within 30 minutes prior to digital thoracic radiography (Figure 1). A minimum of 2-view thoracic radiography was performed to rule-out respiratory disease and only cats without clinical signs of respiratory disease and with radiographically normal lung findings were included. Radiographs were reviewed in DICOM format by board-certified veterinary radiologists who were blinded to lung ultrasound findings. Cats presenting with radiographically diagnosed left-sided CHF (cardiomegaly, left atrial enlargement, pulmonary venous congestion, and interstitial-alveolar infiltrates) and without pleural effusion were similarly enrolled during the same time period as a comparison group analogous to the previously published Vet BLUE study in dogs. ${ }^{4}$

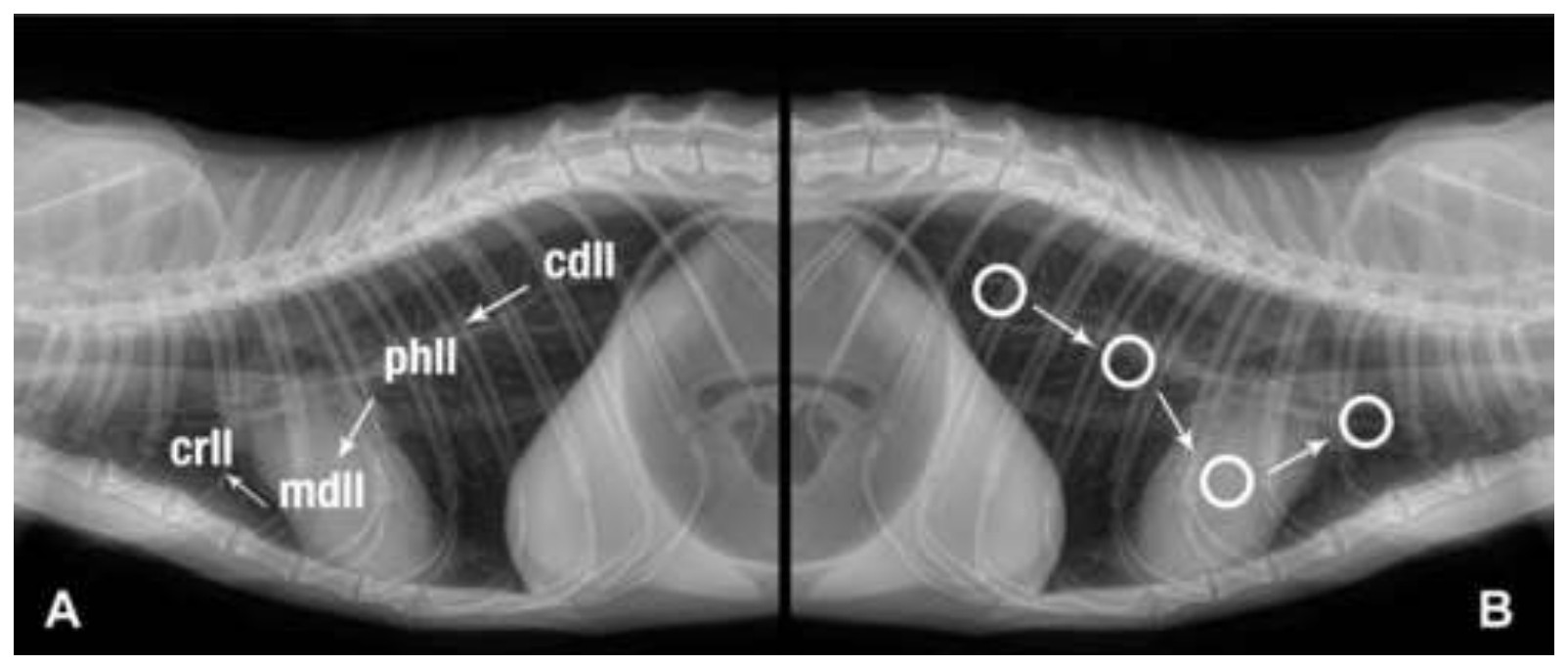

Figure 1. (A) Lateral thoracic radiograph of a cat depicting our study's regionally based lung ultrasound (LUS) views on the left hemithorax by their labeled lung regions: caudodorsal lung region (cdll), perihilar lung region (phll), middle lung region (mdll), and cranial lung region (crll). The LUS regions do not necessarily correlate with anatomically named lung lobes. (B) Circles placed on the mirror image of the same lateral thoracic radiograph in (A) depict the location of ultrasound probe positioning for imaging each respective LUS view. 

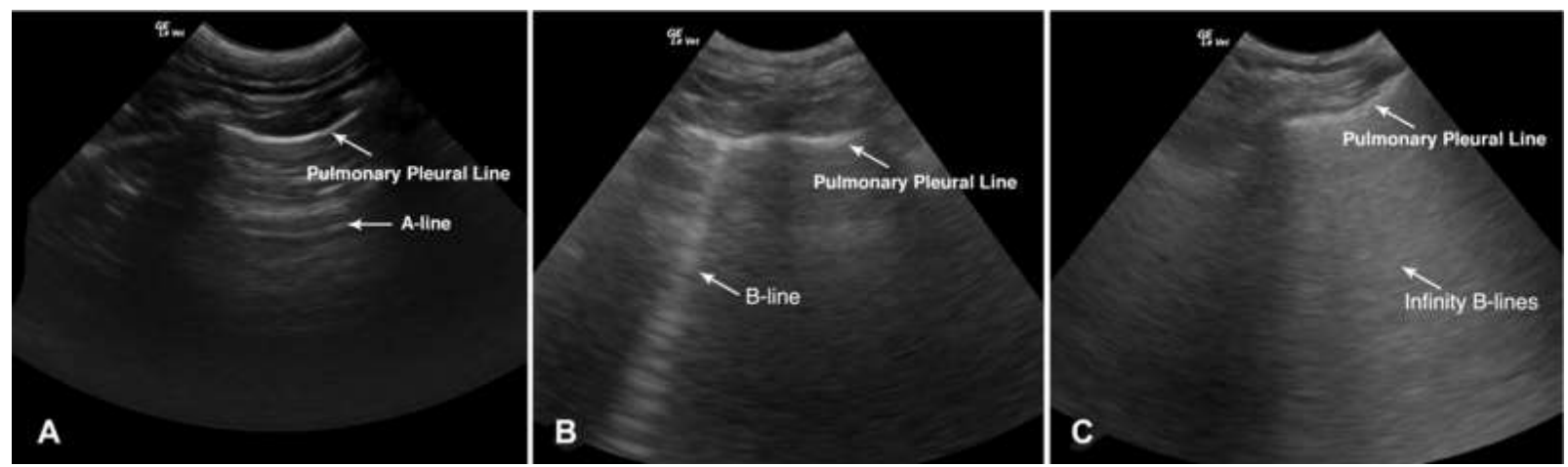

Figure 2. B-mode ultrasound images depicting (A) normal aerated peripheral lung and then degrees of interstitialalveolar edema in the lung periphery as (B) a single B-line and (C) infinity B-lines (or too numerous to count [TNTC]).

The regionally-based LUS protocol (Figure 1) consisting of 8 acoustic windows (4 on each hemithorax: the caudodorsal lung region, perihilar lung region, middle lung region, and cranial lung regions) was performed identically to the previously published Vet BLUE examination in dogs. ${ }^{4}$ Dry lung (A-lines with a glide sign) was defined as, and ultrasonographically recognized by the to and fro motion along the pulmonary-pleural line (glide sign) with air reverberation artifact called A-lines. ${ }^{3,4,12,16}$ A-lines are equidistant parallel lines extending from the pulmonary-pleural line (Figure 2A). ${ }^{1-4,8,12,14}$ Wet lung was defined as, and ultrasonographically recognized by the presence of ULRs also called B-lines (Figure $2 \mathrm{~B}){ }^{1-3,5,6,9-}$ 12,14 ULRs are hyperechoic laser-like streaks that extend from the pulmonary-pleural interface through the far field and oscillate in synchronization with inspiration and expiration while obliterating A-lines. ${ }^{1-3,5,6,9-12,14}$ ULRs are thought to be equivalent to radiographic Kerley B$\operatorname{lines}^{17,18}$ and are associated with interstitial edema in the lung periphery (outer 1-3 mm). ${ }^{17}$ The frequency of ULRs correlates with the degree of pulmonary edema in humans. ${ }^{2,6-8,13,17}$ Additional lung ultrasound findings have been described for the clinical progression of 
interstitial-alveolar disease leading to lung consolidation(shred, tissue and nodule signs) ${ }^{12,14,15}$ but these are unexpected in our study population of cats with radiographically normal lungs.

The maximum number of ULRs over a single intercostal space at each regional lung view was recorded on a standardized data sheet. ULRs were counted in B-mode as follows: 0, none seen; 1, a single discrete ULR; 2, two discrete ULRs; 3, three discrete ULRs; > 3, more than 3 discrete ULRs, and too numerous to count (TNTC also referred to as infinity or white lung) when they blended together (Figure 2C). ${ }^{4,12}$ The standardized data sheet also collected patient identification number, age, sex, breed, body weight, body condition score (9 point scale), investigator and reason for thoracic radiography.

Two sonographers $(\mathrm{XX}, \mathrm{XX})$ performed the lung ultrasound examination studies using the same 8C-RS microconvex, 4-10.0 MHz, ultrasound probe ${ }^{\mathrm{a}}$ but with different ultrasound machines $^{\mathrm{b}, \mathrm{c}}$, Logic e Vet, General Electric (XX) and the Logic XP Note Book (XX), by the same manufacturer (General Electric). ${ }^{\text {b,c }}$ The ultrasound frequency for all examinations was set at 8.0 $\mathrm{MHz}$ and the display depth ranged from $4 \mathrm{~cm}$ to $6 \mathrm{~cm}$. However, a proportion of the left-sided CHF cats had their lung ultrasound examinations performed with a SonoSite Edge ${ }^{\mathrm{d}}{ }^{\mathrm{using}}$ similar ultrasound settings of depth and frequency and a comparable microconvex probe $(\mathrm{C} 11 \mathrm{x}$; 8-5.0 MHz). ${ }^{\mathrm{e}}$ Seven left-sided CHF cats without radiographically evident pleural effusion were consecutively enrolled from a concurrent Vet BLUE respiratory distress study as a clinically relevant comparative cohort.

\section{Statistical Methods}

Categorical data were summarized as frequencies, proportions, and by calculating mid-P exact 95\% confidence intervals (CI). Quantitative data were summarized as medians and inter- 
quartile ranges $\left(25^{\text {th }}\right.$ to $75^{\text {th }}$ percentiles). Categorical data were compared between groups of cats using chi-square and Fisher exact tests. Quantitative data were compared across groups using Mann-Whitney U tests. Observed ULRs were compared between left and right sides using Wilcoxon signed-rank tests. For statistical analysis, reasons for thoracic radiography were categorized as clinically healthy, trauma, metabolic conditions, and other reasons. Categorical data analysis and quantitative data were analysed using commercially available software. ${ }^{\mathrm{f}, \mathrm{g}}$ Results were interpreted at the $5 \%$ level of significance.

\section{Results}

Data from 49 cats without clinical signs of respiratory disease and with radiographically normal lungs were collected during the study period. Cats presented for trauma $(\mathrm{n}=12)$, various metabolic conditions $(n=15)$, and for other reasons $(n=12)$. Ten clinically healthy cats were also enrolled. The study population consisted of domestic shorthair $(\mathrm{n}=34)$, domestic medium/long hair $(n=7)$, and other breeds $(n=8)$. There were 15 spayed females, 29 neutered males, 3 intact females, and 2 intact males. The median (range) age, weight, and body condition score were 5 years $(1,13), 4.3 \mathrm{~kg}(3.4,6.1)$, and $5(4,6)$, respectively (Table 1). One investigator (XX) performed $67 \%$ (33/49) of the lung ultrasound examinations and the other performed the remainder (XX). Thoracic radiographs consisted of the minimum of 2-views (one right or left lateral view plus one ventrodorsal or dorsoventral view) per patient (47/49) and 2 cats were evaluated by 3-view thoracic radiography (right and left lateral views plus one ventrodorsal or dorsoventral view). Seven cats with radiographically-evident left-sided CHF without radiographically-evident pleural effusion were enrolled during the study period as a comparison group. Within the left-sided CHF cohort, there were 3 spayed females and 4 neutered males 
which included 6 domestic shorthair and 1 Burmese. The median (range) age, weight, and body condition score were 13 years $(3,20), 4.9 \mathrm{~kg}(2.5,6.0)$, and $4.5(2,5.5)$, respectively.

Table 1. Frequency of B-lines based on specific lung regions within 49 cats without respiratory disease and 7 cats with left-sided congested heart failure (CHF) enrolled from 3 emergency referral practices

\section{Normal cats $\quad$ Left-sided CHF cats}

Side Lung region Max Proportion (95\% CI) Max Proportion (95\% CI) $P$ value*

$\begin{array}{ccllll}\text { Left } \begin{array}{ccc}\text { Dorsal } \\ \text { Perihilar }\end{array} & 2 & 0.00(0,0.06) & 0.02(0,0.10) & \text { TNTC } 0.86(0.47,0.99) & <0.001 \\ \text { Middle } & 1 & 0.02(0,0.10) & \text { TNTC } 0.86(0.47,0.99) & <0.001 \\ \text { Cranial } & 1 & 0.02(0,0.10) & \text { TNTC } 0.86(0.47,0.99) & <0.001 \\ \text { Sum (all) } & 2 & 0.06(0.02,0.16) & \text { TNTC } 1.0(0.65,1.0) & <0.001 \\ \text { Right Dorsal } & 1 & 0.04(0.01,0.13) & >3 & 0.50(0.15,0.85) & 0.093 \\ \text { Perihilar } & 0 & 0(0,0.06) & \text { TNTC } 0.86(0.47,0.99) & <0.001 \\ \text { Middle } & 1 & 0.02(0,0.10) & \text { TNTC } 0.86(0.47,0.99) & <0.001 \\ \text { Cranial } & 1 & 0.02(0,0.10) & \text { TNTC } 0.86(0.47,0.99) & <0.001 \\ \text { Sum (all) } & 1 & 0.08(0.03,0.19) & \text { TNTC } 1.0(0.65,1.0) & <0.001 \\ \text { Sum (all) } & 2 & 0.12(0.05,0.24) & \text { TNTC } 1.0(0.65,1.0) & <0.001\end{array}$

*Based on Mann-Whitney U tests.

TNTC, too numerous to count. 
Overall frequency of ULRs was $12 \%$ (95\% CI, 5-24\%) in cats without clinical signs of respiratory disease and 100\% (95\% CI, 65-100\%) in cats with left-sided heart failure (Table 1). The majority of cats without respiratory disease and with ULRs had lung rockets observed at a single location (5/6). The sixth cat had 2 positive sites with only a single ULR at each respective site. Of the 5 cats with a single ULR-positive site, only a single ULR was observed in 4 of 5 with the other cat having 2 ULRs at its single positive site. No significant predictors were identified for the presence of ULRs in cats with radiographically normal lungs (Table 2). All of the cats with left-sided CHF (7/7) had >3 URLs observed at every Vet BLUE regionally-based view (Table 2).

Table 2. Comparison of factors between cats with B-lines and those without within 49 cats without respiratory disease enrolled from three emergency referral practices

Overall B-lines No B-lines

Variable PE (n) $95 \%$ CI or range* PE (n) 95\% CI or range* PE (n) 95\% CI or range* $P$ value $\stackrel{+}{*}$

Presenting complaint

$\begin{array}{llll}\text { Metabolic } 0.31(15) & 0.19,0.44 & 0.33(2) 0.06,0.74 & 0.30(13) 0.18,0.45 \quad 1.0\end{array}$



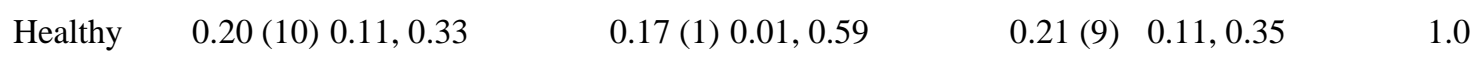

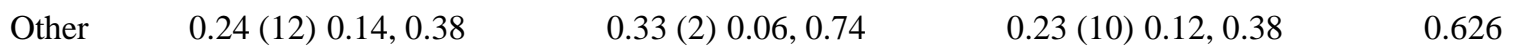

Sex

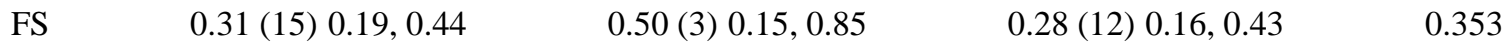



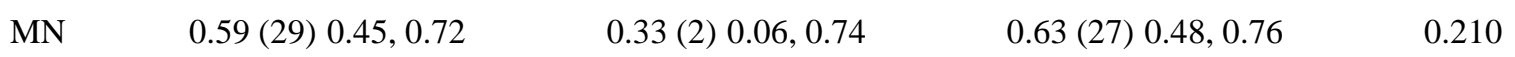



Overall
B-lines
No B-lines



\section{Discussion}

Overall frequency and number of detected ULRs using Vet BLUE was low at $12 \%$ for radiographically normal lungs in cats with dry lung artifacts predominating similar to previous 
findings in an analogous Vet BLUE study in dogs. ${ }^{4}$ In contrast, the comparison group of 7 cats with left-sided CHF had a higher frequency (100\%) and larger number of ULRs (wet lung artifacts) observed per positive lung region. These results are consistent with the presence of pulmonary edema similar to findings in dogs and humans. ${ }^{1,4,5,9,10}$ The number and frequency of ULRs has been used to reliably rule-in or rule-out left-sided CHF in people ${ }^{1,2,8,10,13,18}$ The results of this study and previous studies in dogs document repeatable similarities to the clinical course and ultrasonographic appearance of pulmonary interstitial disease in humans. ${ }^{3-5,11}$ In other words, the finding of dry lung artifacts in all Vet BLUE fields likely rules out any clinically relevant left-sided CHF in cats as has been shown in humans. ${ }^{1,5-10}$

No factors were identified that were significant predictors of the presence of ULRs in the cats with radiographically normal lungs. This finding is in contrast to that of dogs in which neutered males had a higher frequency of single positive sites; however, it was concluded that this may have been due to the small sample size or for reasons for radiographic evaluation, i.e. more trauma was present in the study population of neutered male dogs. ${ }^{4}$ A specific Vet BLUE lung region, positive for ULRs or B-lines, was not found to be statistically significant despite the logic that the less aerated, more perfused, most ventral lung lobes should have a higher frequency of ULRs or B-lines than the more aerated, least perfused, most dorsal lung fields. This finding in cats was also found in an analogous study in dogs. ${ }^{4}$

There were some limitations to our clinical study. The prospective study reported here enrolled cats that were free of respiratory disease for the evaluation of the normal frequency of wet lung artifacts referred to as ULRs (B-lines). However, these cats were not necessarily healthy cats since many presented for other illnesses. On the other hand, the use of this subset of felines may make the established baseline frequency of ULRs even more clinically valuable 
since our study documents a low frequency in ill cats without respiratory signs and with radiographically normal lungs. Another limitation involved the use of 2-view rather than 3-view thoracic radiography which occurred primarily because of the difference between levels of tolerance of restraint (cats being less tolerant than dogs); and no cats were sedated for examination in this study. In a similarly designed dog study, no statistical difference was found among the dogs having 2-view versus 3 -view thoracic radiographic studies. ${ }^{4}$ However, it is welldocumented that the identification of a lung mass or infiltrate is enhanced by obtaining left and right lateral recumbent views because lung lesions are obscured due to compression and atelectasis in the dependent lung parenchyma. ${ }^{19-21}$ Eliminating one view from a 3-view study has been shown to change patient diagnoses ${ }^{22}$ and 2-view exams comprised of only left and right lateral recumbency are the most sensitive 2-view combination for lung masses. ${ }^{23}$ In contrast to human patients, the shape of the canine and feline thorax causes the dorsal and ventral aspects of lung fields to silhouette with soft tissue opacity structures along midline in dorsoventral and ventrodorsal views and pulmonary edema in cats has a random, patchy distribution rather than distinct perihilar focus. ${ }^{24}$ For these reasons, 3-view thoracic exams remain the radiographic standard for detection of interstitial lung pathology and clinicians must weigh these factors when determining the diagnostic plan for their patients.

The comparative findings to left-sided CHF cats appear to support our hypothesis that wet lung artifacts represented by ULRs (B-lines) will be more frequent and found in higher numbers in cats with interstitial-alveolar lung edema. A larger sample size would be necessary to make valid inferences but the same lung ultrasound imaging principles and limitations described for human patients are applicable to canine and feline patients. First, identification of abnormal lung ultrasound findings relies on respiratory pathology that extends to the outermost 
lung tissue since ultrasound waves do not transmit through normal, aerated lung. In most acute conditions, lung ultrasound findings commonly include ULRs (B-lines), which often represent interstitial-alveolar edema; however, other lung ultrasound findings of interstitial-alveolar consolidation are possible. ${ }^{1,2,5,6-8,14,17,18,25,26}$ In contrast, more isolated disease states such as masses (neoplastic, granulomatous, infectious), consolidation (alveolar lung pattern), and early interstitial diseases may not initially extend to the lung periphery and thus are missed by lung ultrasound. ${ }^{1,25-8,14-18,25,26,27}$ However, it has been shown that many acute forms of interstitial and alveolar lung pathology in human patients and animals do in fact extend to the lung periphery and are readily recognized by lung ultrasound. ${ }^{1,2,5,8,14,15,17,18,25-27,29}$ The size and shape of the feline thorax requires detailed interpretation and interpolation of patient radiographic, ultrasonographic and physical exam findings to achieve the greatest diagnostic accuracy. In conclusion, ultrasound lung rockets (B-lines) are easily recognizable and quantifiable lung ultrasound artifacts which serve as an objective evaluation unaffected by environmental and patient noise or auscultation skills. ${ }^{3-5,9,10,29}$ The low frequency and number of ULRs in cats without clinical signs of respiratory disease and with radiographically normal lung findings suggest that the use of a standardized, pattern-based approach like Vet BLUE will be clinically useful for the identification of cats with certain respiratory conditions. 


\section{Footnotes}

${ }^{a}$ Ultrasound Probe, Microconvex Model 8C-RS, General Electric, Milwaukee, Wisconsin.

${ }^{\mathrm{b}}$ Logic e Vet, General Electric, Milwaukee, Wisconsin.

${ }^{\mathrm{c}}$ Logic XP Note Book, General Electric, Milwaukee, Wisconsin.

${ }^{\mathrm{d}}$ SonoSite Edge ${ }^{\circledR}$, Bothell, Washington.

${ }^{\mathrm{e}}$ Ultrasound Probe, Micronconvex Model C11x, SonoSite, Bothell, Washington.

${ }_{\mathrm{f}}^{\mathrm{f}}$ Epi Info, Version 6.04, Center for Disease Control, Atlanta, Georgia.

${ }^{\mathrm{g}}$ IBM SPSS Statistics, Version 21, SPSS Inc, Chicago, Illinois.

\section{References}

1. Lichtenstein DA, Meziere GA. Relevance of lung ultrasound in the diagnosis of acute respiratory failure: the BLUE protocol. Chest 2008;134(1):117-25.

2. Volpicelli G, Elbarbary $\mathrm{M}$, Blaivas $\mathrm{M}$, et al. International evidence-based recommendations for point-of-care lung ultrasound. Intensive Care Med 2012;38:577-91.

3. Lisciandro GR. Abdominal and thoracic focused assessment with sonography for trauma, triage, and monitoring in small animals. J Vet Emerg Crit Care 2011;21(2):104-22.

4. Lisciandro GR, Fosgate GT, Fulton RM. Frequency Of Ultrasound Lung Rockets Using A Regionally-based Lung Ultrasound Examination Named Veterinary Bedside Lung Ultrasound Exam (Vet BLUE) In 98 Dogs With Normal Thoracic Radiographical Lung Findings. Vet Rad \& US 2014;55(3):315-22.

5. Rademacher N, Pariaut R, Pate J, et al. Transthoracic lung ultrasound in normal dogs and dogs with cardiogenic pulmonary edema: A pilot study. Vet Rad \& US 2014;55(4):447-452. 
6. Lichtenstein D, Karakitsos D. Integrating lung ultrasound in the hemodynamic evaluation of acute circulatory failure (the fluid administration limited by lung sonography protocol). J Crit Care 2012;27(5):533.

7. Lichtenstein D. Fluid administration limited by lung sonography: the place of lung ultrasound in assessment of acute circulatory failure (the FALLS-protocol). Expert Rev Respir Med 2012;6(2):155-62.

8. Lichtenstein DA, Meziere GA, Lagoueyte J, et al. A-Lines and B-Lines. Lung ultrasound as a bedside tool for predicting pulmonary artery occlusion pressure in the critically ill. Chest 2009;136(4):1014-20.

9. Liteplo AS, Marill KA, Villen T, et al. Emergency thoracic ultrasound in the differentiation of the etiology of shortness of breath (ETUDES): Sonographic B-lines and Nterminal pro-brain-type natriuretic peptide in diagnosing congestive heart failure. Acad Emerg Med 2009;16:201-210.

10. Kobal SL, Trento L, Baharami S, et al. Comparison of effectiveness of hand-carried ultrasound to bedside cardiovascular physical examination. Am J Cardiol 2005;96:1002-1006.

11. Boysen SR, Lisciandro GR. The use of ultrasound for cats and cats in the emergency room: AFAST and TFAST. Vet Clin North Am Small Anim Pract. 2013;43(4):773-97.

12. Lisciandro GR. The Vet BLUE Lung Scan. In: Lisciandro GR, ed. Focused Ultrasound Techniques for the Small Animal Practitioner. Ames IA; Wiley-Blackwell, 2014;166-188.

13. Lichtenstein DA. Ultrasound examination of the lungs in the intensive care unit. Pediatr Crit Care Med. 2009;10(6):693-8

14. Lichtenstein D. Should lung ultrasonography be more widely used in the assessment of acute respiratory disease? Expert Rev Respir Med 2010;4(5):533-38. 
15. Lichtenstein DA, Lascos N, Meziere GA, Gelper A. Ultrasound diagnosis of alveolar consolidation in the critically ill. Intensive Care Med 2004;30:276-281.

16. Lisciandro GR, Lagutchik MS, Mann KA, et al. Evaluation of a thoracic focused assessment with sonography for trauma (TFAST) protocol to detect pneumothorax and concurrent thoracic injury in 145 traumatized cats. J Vet Emerg Crit Care 2008;18(3):258-269.

17. Soldati G, Sher S, Testa A. Lung and ultrasound: time to "reflect." Eur Rev Med Pharmacol Sci 2011;15(2):223-7.

18. Lichtenstein DA. Ultrasound in the management of thoracic disease. Crit Care Med 2007; 35(S5):S250-61.

19. Thrall DE. Principles of Radiographic Interpretation of the Thorax. In: Thrall DE, ed. Textbook of Veterinary Diagnostic Radiology, $6^{\text {th }}$ edition, St Louis MO: Elsevier 2013;474-478.

20. Penchman RD. Effect of dependency vs. non-dependency on lung lesion visualization. Vet Radiol 1987;28:185-190.

21. 20. Spencer CP, Ackerman N, Burt JK. The canine lateral thoracic radiograph. Vet Radiol 1981;22:262-266.

22. Ober CP, Barber D. Comparison of two- vs. three-view thoracic radiographic studies on conspcuity of structured interstitial patterns in dogs. Vet Radiol \& US 2006; 47(6):542-545.

23. Lang J, Worthman JA, Glickman LT, et al. Sensitivity of radiographic detection of lung metastasis in the dog. Vet Radiol \& US 1986;27:74-78.

24. Thrall DE. The Thoracic Cavity: Canine, Feline, and Equine. In: Thrall DE, ed. Textbook of Veterinary Diagnostic Radiology, $6^{\text {th }}$ edition, St Louis MO: Elsevier 2013; pp. 596. 
25. Gargani L, Lionetti V, Di Cristofano C, et al. Early detection of acute lung injury uncoupled to hypoxemia in pigs using ultrasound lung comets. Crit Care Med 2007; 35(12):2769-2774.

26. Peris A, Zagli G, Barbani F, et al. The value of lung ultrasound monitoring in H1N1 acute respiratory distress syndrome. Anaesthesia 2010;65(3):294-7.

27. Soldati G, Testa A, Silva FR, et al. Chest ultrasonography in lung contusion. Chest 2006;130(2):533-8.

28. Filly RA. Ultrasound: the stethoscope of the future, alas. Radiology 1988;167:400.

29. Buczinski S, Forte G, Francoz D, et al. Comparison of thoracic auscultation, clinical score and ultrasonography, as indicators of bovine respiratory disease in preweaned diary calves. J Vet Intern Med 2014;28:234-42. 